\title{
Characterizing Variable Memory Property of the Smoking Habit Model
}

\author{
Amaal Sayed \\ Department of Mathematics, Faculty of Engineering, October University for Modern Sciences and Arts \\ (MSA), Giza, Egypt.E-mail: amal_wa84@yahoo.com
}

\section{Khalil}

Department of Mathematics, Faculty of Engineering, October University for Modern Sciences and Arts (MSA), Giza, Egypt. E-mail: mkibrahim@msa.eun.eg

\author{
A.M.A. Arafa \\ Department of Mathematics, Faculty of Science, Port Said University, Port Said, Egypt \\ E-mail: anas_arafa@sci.psu.edu.eg
}

Received: 10 Oct. 2019 / Accepted 05 Dec. 2019 / Publication date: 30 Dec. 2019

\begin{abstract}
Fractional calculus is one of the best mathematical tools to characterize the memory property of the dynamical systems. So, Variable-order fractional derivatives in Caputo sense are used in characterizing the memory property of the presented dynamic smoking habit model. Numerical solutions are obtained using a predictor-corrector method to handle the fractional derivatives. These numerical solutions show that the modeling of smoking habit model with variable-order fractional derivatives has more advantaged than integer-order derivatives.
\end{abstract}

Keywords: Smoking habit model, Fractional calculus, dynamical systems, Predictor-corrector method, and Variable-order fractional derivatives.

\section{Introduction}

The memory property has been found in complex systems (Guerrero et al., 2011). Characterizing the memory property of systems is a challenging issue in phenomenological description. So, fractional calculus is used to characterize the memory property of complex systems (Turski et al., 2002). The variable-order fractional derivative has the significant feature of capturing the history of the variable which cannot be easily done in case of the integer order derivative (Pinto and Machado, 2013). The variable-order fractional derivatives has been presented in many researches (El-Sayed et al., 2016; ElSayed et al., 2017; Khalil et al., 2018; Arafa et al., 2019) which is good at depicting the memory property which changes with time.

These days, smoking habit is one of the main health problems in the world. More than 4 million deaths are caused due to the effect of smoking worldwide. Recently, there are several diseases such as throat cancer, lung cancer, heart disease, and mouth cancer which are the main threatening due to longterm smoking. Mathematicians tried to make different effective smoking models which proposed by several authors; for example the optimal campaign in the smoking dynamical system is analyzed in (Zaman, 2011). The mathematical description of the dynamics of tobacco use, recovery and relapse is examined by Garsow et al. (1997).

From mathematicians' perspective, mathematical models are significant tools that help us to understand the current state and the future progress of diseases in human networks in order to control and prevent such diseases (Arafa et al., 2019).

This paper is organized as follows. In Section 2, some preliminaries of fractional calculus, the algorithm of the predictor-corrector method are presented, and the proposed model. In Section 3 is devoted to the numerical results and discussions. Our conclusion is illustrated in Section 4.

Corresponding Author: Amaal Sayed, Department of Mathematics, Faculty of Engineering, October University for modern sciences and arts (MSA), Giza, Egypt. E-mail: amal_wa84@yahoo.com 


\section{Materials and Methods}

\section{Preliminaries}

We present some basic definitions of variable fractional-order derivatives as follows:

Definition 1 (Riemann-Liouville derivatives of variable-order fractional $\alpha(t)$ )

Let $\alpha(t)$ be a bounded and continuous function, then Riemann-Liouville variable-order fractional derivative of $f(t):[a, b] \rightarrow \mathbb{R}$ is defined as (Xu and He, 2013):

i) Left Riemann-Liouville derivative of variable-order fractional $\alpha(t)$ is defined by

$$
{ }_{a}^{R L} D_{t}^{\alpha(t)} f(t)=\frac{1}{\Gamma(1-\alpha(t))} \frac{d}{d t} \int_{a}^{t}(t-\omega)^{-\alpha(t)} f(\omega) d \omega, \quad 0<\alpha(t) \leq 1
$$

ii) Right Riemann-Liouville derivative of variable-order fractional $\alpha(t)$ is defined by

$$
{ }_{t}^{R L} D_{b}^{\alpha(t)} f(t)=\frac{1}{\Gamma(1-\alpha(t))} \frac{d}{d t} \int_{t}^{b}(t-\omega)^{-\alpha(t)} f(\omega) d \omega, \quad 0<\alpha(t) \leq 1
$$

Definition 2 (Caputo derivatives of variable-order fractional $\alpha(t)$ )

Let $\alpha(t)$ be a bounded and continuous function, then the Caputo variable-order fractional derivative of $f(t):[a, b] \rightarrow \mathbb{R}$ is defined as (Xu and He, 2013):

i) Left Caputo derivative of variable-order fractional $\alpha(t)$ is defined by

$$
{ }_{a}^{C} D_{t}^{\alpha(t)} f(t)=\frac{1}{\Gamma(1-\alpha(t))} \int_{a}^{t}(t-\omega)^{-\alpha(t)} f^{\prime}(\omega) d \omega, \quad 0<\alpha(t) \leq 1
$$

ii) Right Caputo derivative of variable-order fractional $\alpha(t)$ is defined by

$$
{ }_{t}^{C} D_{b}^{\alpha(t)} f(t)=\frac{-1}{\Gamma(1-\alpha(t))} \int_{t}^{b}(t-\omega)^{-\alpha(t)} f^{\prime}(\omega) d \omega, \quad 0<\alpha(t) \leq 1
$$

\section{The model derivation}

The following model has been used successfully to predict the evolution of the prevalence of smoking in Spain in integer order (Guerrero et al., 2011). We convert that model into variable-order fractional model which consists of four types of individuals, whose proportions are defined by $\mathrm{x}$ (nonsmokers), y (normal smokers), z (excessive smokers) and w (ex-smokers) as follows:

$$
\begin{aligned}
& { }^{C} D^{\alpha_{1}(t)} x(t)=\mu(1-x(t))-\beta x(t)(y(t)+z(t)), \\
& { }^{C} D^{\alpha_{2}(t)} y(t)=\beta x(t)(y(t)+z(t))+\rho w(t)+a z(t)-(\lambda+\gamma+\mu) y(t), \\
& { }^{C} D^{\alpha_{3}(t)} z(t)=y(t)-(a+\delta+\mu) z(t), \\
& { }^{C} D^{\alpha_{4}(t)} w(t)=\lambda y(t)+\delta z(t)-(\rho+\mu) w(t) .
\end{aligned}
$$


The parameters of the model are

- $\mu$ is birth rate in Spain.

- $\beta$ is transmission rate due to social pressure to adopt smoking habit.

- $\rho$ is rate at which ex-smokers return to smoking.

- $a$ is rate at which an excessive smoker becomes a normal smoker by decreasing the number of cigarettes per day.

- $\gamma$ is rate at which normal smokers become excessive smokers by increasing the number of cigarettes per day.

- $\lambda$ is rate at which normal smokers stop smoking.

- $\delta$ is rate at which excessive smokers stop smoking.

Since the constant population has been normalized to unity, our variables satisfy that

$$
x(t)+y(t)+z(t)+w(t)=1 .
$$

\section{The predictor-corrector method}

There are many techniques for solving a variable-order fractional model. We state a predictor-corrector method for solving a variable-order fractional model.

We will introduce an algorithm of predictor-corrector method for solving the following system of variable-order fractional differential equations

$$
\begin{aligned}
& D^{\alpha_{1}(t)} x(t)=f_{1}(x(t), y(t), z(t), w(t)), \\
& D^{\alpha_{2}(t)} y(t)=f_{2}(x(t), y(t), z(t), w(t)), \\
& D^{\alpha_{3}(t)} z(t)=f_{3}(x(t), y(t), z(t), w(t)), \\
& D^{\alpha_{4}(t)} w(t)=f_{4}(x(t), y(t), z(t), w(t)),
\end{aligned}
$$

With $0<\alpha_{i}(t) \leq 1(i=1,2,3,4)$ and initial conditions $\left(x_{0}, y_{0}, z_{0}, w_{0}\right)$.

Evaluate the predicted values as follows: 


$$
\begin{aligned}
& x_{n+1}^{p}=x_{0}+\sum_{j=0}^{n} \frac{\beta_{1, j, n+1}}{\Gamma\left(\alpha_{1}\left(t_{n+1}\right)\right)} f_{1}\left(x_{j}, y_{j}, z_{j}, w_{j}\right), \\
& y_{n+1}^{p}=y_{0}+\sum_{j=0}^{n} \frac{\beta_{2, j, n+1}}{\Gamma\left(\alpha_{2}\left(t_{n+1}\right)\right)} f_{2}\left(x_{j}, y_{j}, z_{j}, w_{j}\right), \\
& z_{n+1}^{p}=z_{0}+\sum_{j=0}^{n} \frac{\beta_{3, j, n+1}}{\Gamma\left(\alpha_{3}\left(t_{n+1}\right)\right)} f_{3}\left(x_{j}, y_{j}, z_{j}, w_{j}\right), \\
& w_{n+1}^{p}=w_{0}+\sum_{j=0}^{n} \frac{\beta_{4, j, n+1}}{\Gamma\left(\alpha_{4}\left(t_{n+1}\right)\right)} f_{4}\left(x_{j}, y_{j}, z_{j}, w_{j}\right),
\end{aligned}
$$

Where

$$
\beta_{i, j, n+1}=\frac{h^{\alpha_{i}\left(t_{n+1}\right)}}{\alpha_{i}\left(t_{n+1}\right)}\left[(n-j+1)^{\alpha_{i}\left(t_{n+1}\right)}-(n-j)^{\alpha_{i}\left(t_{n+1}\right)}\right] \cdot h=T / N, T_{n}=n h .
$$

Evaluate the corrected values as follows

$$
\begin{aligned}
& x_{n+1}=x_{0}+\frac{h^{\alpha_{1}\left(t_{n+1}\right)}}{\Gamma\left(\alpha_{1}\left(t_{n+1}\right)+2\right)}+f_{1}\left(x_{n+1}^{p}, y_{n+1}^{p}, z_{n+1}^{p}, w_{n+1}^{p}\right)+\sum_{j=0}^{n} \frac{h^{\alpha_{1}\left(t_{n+1}\right)} \gamma_{1, j n+1}}{\Gamma\left(\alpha_{1}\left(t_{n+1}\right)+2\right)} f_{1}\left(x_{j}, y_{j}, z_{j}, w_{j}\right), \\
& y_{n+1}=y_{0}+\frac{h^{\alpha_{2}\left(t_{n+1}\right)}}{\Gamma\left(\alpha_{2}\left(t_{n+1}\right)+2\right)}+f_{2}\left(x_{n+1}^{p}, y_{n+1}^{p}, z_{n+1}^{p}, w_{n+1}^{p}\right)+\sum_{j=0}^{n} \frac{h^{\alpha_{2}\left(t_{n+1}\right)} \gamma_{2, j, n+1}}{\Gamma\left(\alpha_{2}\left(t_{n+1}\right)+2\right)} f_{2}\left(x_{j}, y_{j}, z_{j}, w_{j}\right), \\
& z_{n+1}=z_{0}+\frac{h^{\alpha_{3}\left(t_{n+1}\right)}}{\Gamma\left(\alpha_{3}\left(t_{n+1}\right)+2\right)}+f_{3}\left(x_{n+1}^{p}, y_{n+1}^{p}, z_{n+1}^{p}, w_{n+1}^{p}\right)+\sum_{j=0}^{n} \frac{h^{\alpha_{3}\left(t_{n+1}\right)} \gamma_{3, j, n+1}}{\Gamma\left(\alpha_{3}\left(t_{n+1)}\right)+2\right)} f_{3}\left(x_{j}, y_{j}, z_{j}, w_{j}\right), \\
& w_{n+1}=w_{0}+\frac{h^{\alpha_{4}\left(t_{n+1}\right)}}{\Gamma\left(\alpha_{3}\left(t_{n+1}\right)+2\right)}+f_{4}\left(x_{n+1}^{p}, y_{n+1}^{p}, z_{n+1}^{p}, w_{n+1}^{p}\right)+\sum_{j=0}^{n} \frac{h^{\alpha_{4}\left(t_{n+1}\right)} \gamma_{4, j, n+1}}{\Gamma\left(\alpha_{4}\left(t_{n+1)}\right)+2\right)} f_{4}\left(x_{j}, y_{j}, z_{j}, w_{j}\right),
\end{aligned}
$$

where

$$
\gamma_{i, j, n+1}= \begin{cases}n^{\alpha_{i}\left(t_{n+1}\right)+1}-\left(n-\alpha_{i}\left(t_{n+1}\right)\right)(n+1)^{\alpha_{i}\left(t_{n+1}\right)} & , j=0, \\ (n-j-2)^{\alpha_{i}\left(t_{n+1}\right)+1}+(n-j)^{\alpha_{i}\left(t_{n+1}\right)+1}-2(n-j+1)^{\alpha_{i}\left(t_{n+1}\right)} & , \quad 1 \leq j \leq n, \\ 1 & , j=n+1\end{cases}
$$




\section{Results and Discussion}

We applied the predictor-corrector method to get the numerical solution of the system (5) with the values of the parameters

$\mu=0.01, \rho=0.0425, \beta=0.0381, a=0.1244, \gamma=0.1175, \lambda=0.0498, \delta=0.0498$.

and initial conditions $x(0)=0.5045, y(0)=0.059, z(0)=0.1559, w(0)=0.1337$.

we investigate the system behavior in two cases. First case when the variable-order fractional is $\alpha(t)=0.8-0.005 t$. Second case when the variable-order fractional $\alpha(t)=0.8-0.05 \sin (\pi t)$.

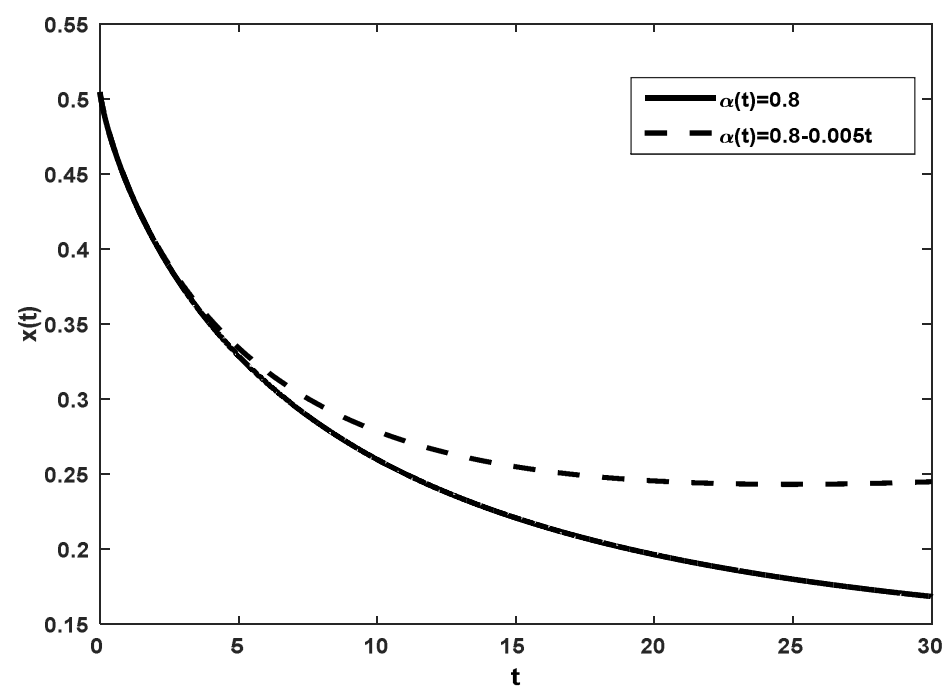

Fig. 1: The solution of $\mathrm{x}(\mathrm{t})$ at $\alpha(t)=0.8$ (the solid line) and at $\alpha(t)=0.8-0.005 t$ (dashed line).

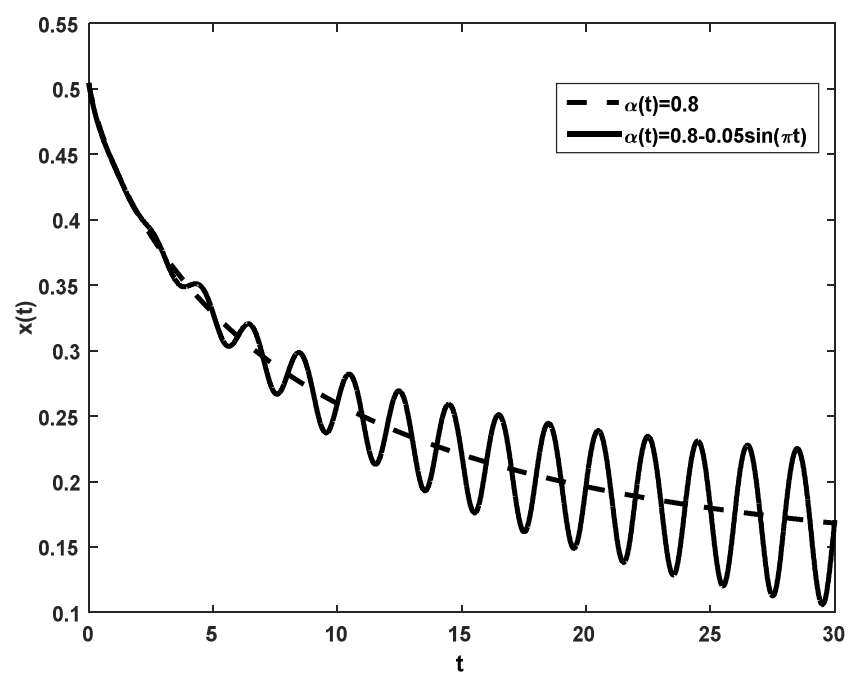

Fig. 2: The solution of $\mathrm{x}(\mathrm{t})$ at $\alpha(t)=0.8$ (the dashed line) and at $\alpha(t)=0.8-0.05 \sin (\pi t)$ (solid line). 


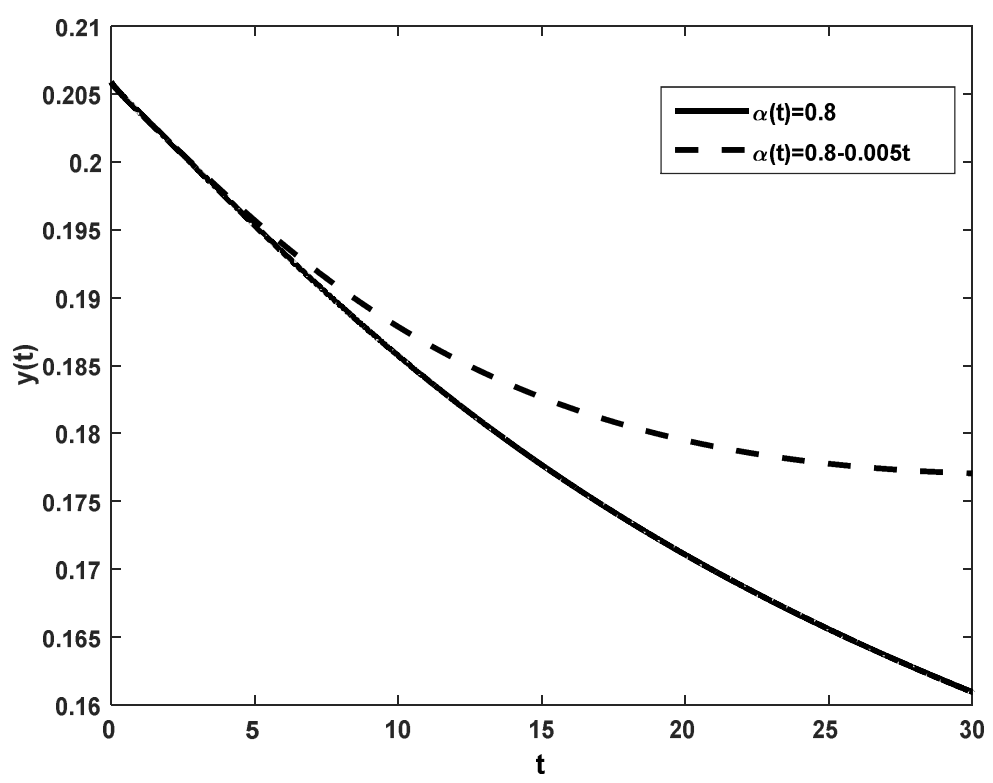

Fig. 3: The solution of $\mathrm{y}(\mathrm{t})$ at $\alpha(t)=0.8$ (the solid line) and at $\alpha(t)=0.8-0.005 t$ (dashed line).

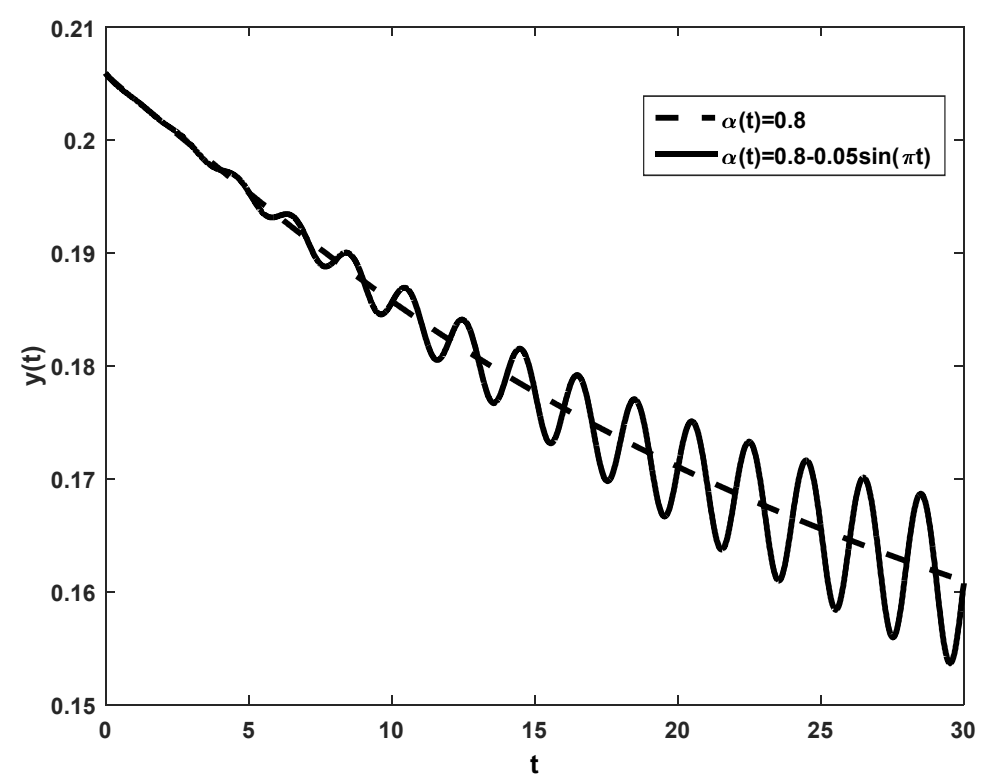

Fig. 4: The solution of $\mathrm{y}(\mathrm{t})$ at $\alpha(t)=0.8$ (the dashed line) and at $\alpha(t)=0.8-0.05 \sin (\pi t)$ (solid line). 


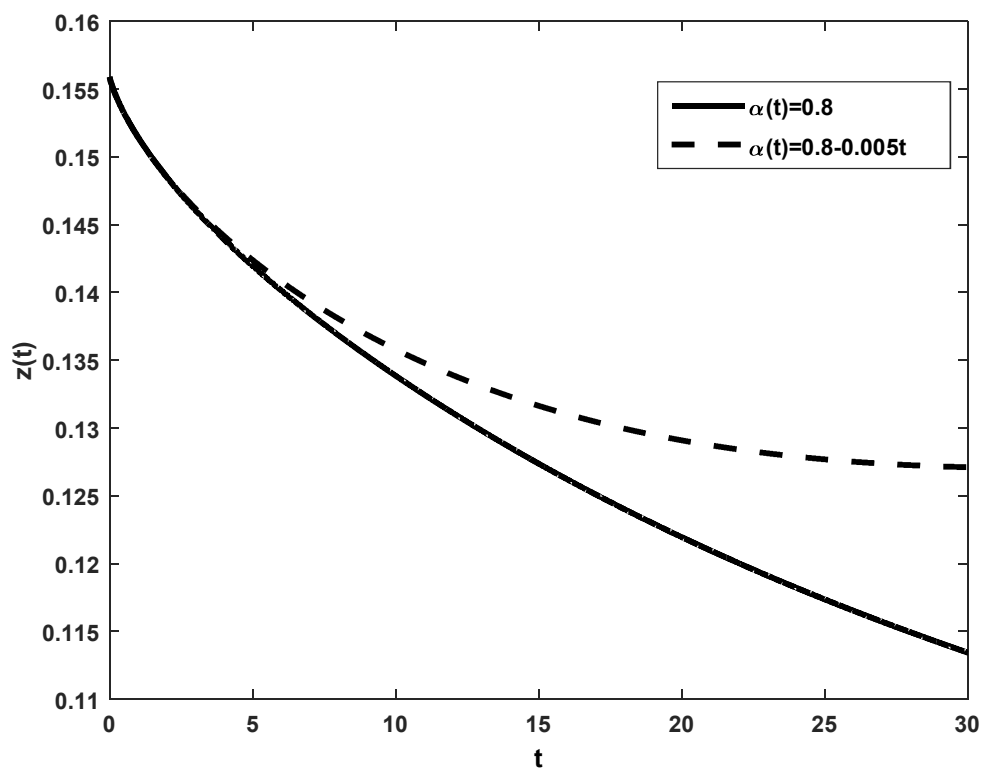

Fig. 5: The solution of $\mathrm{z}(\mathrm{t})$ at $\alpha(t)=0.8$ (the solid line) and at $\alpha(t)=0.8-0.005 t$ (dashed line).

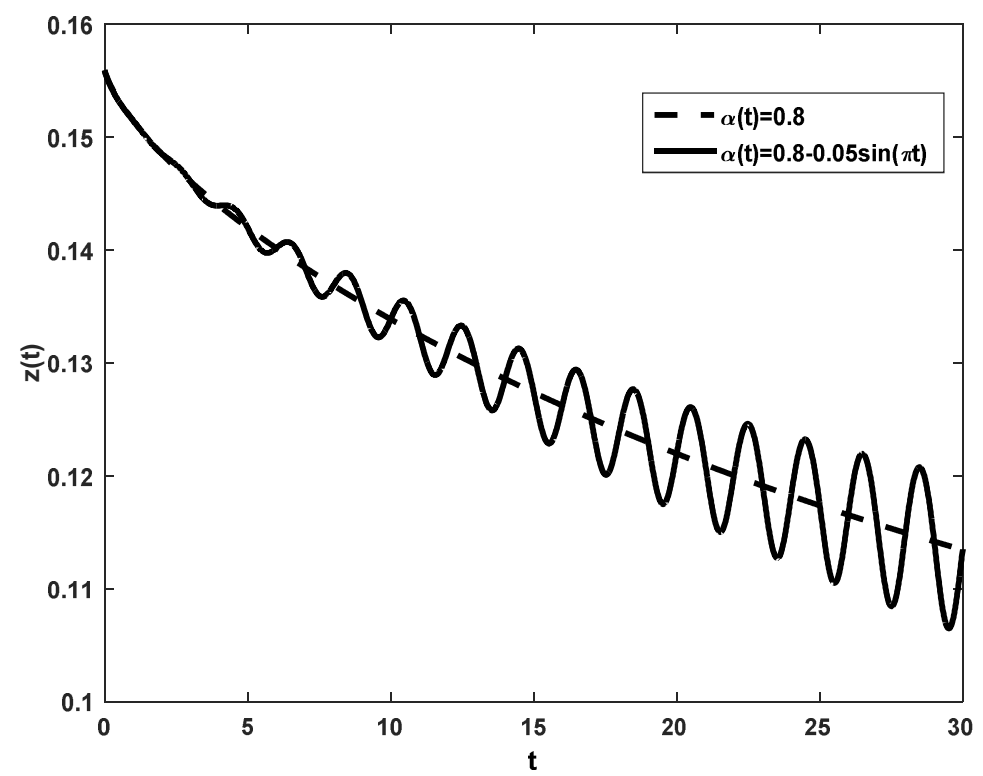

Fig. 6: The solution of $z(t)$ at $\alpha(t)=0.8$ (the dashed line) and at $\alpha(t)=0.8-0.05 \sin (\pi t)$ (solid line). 


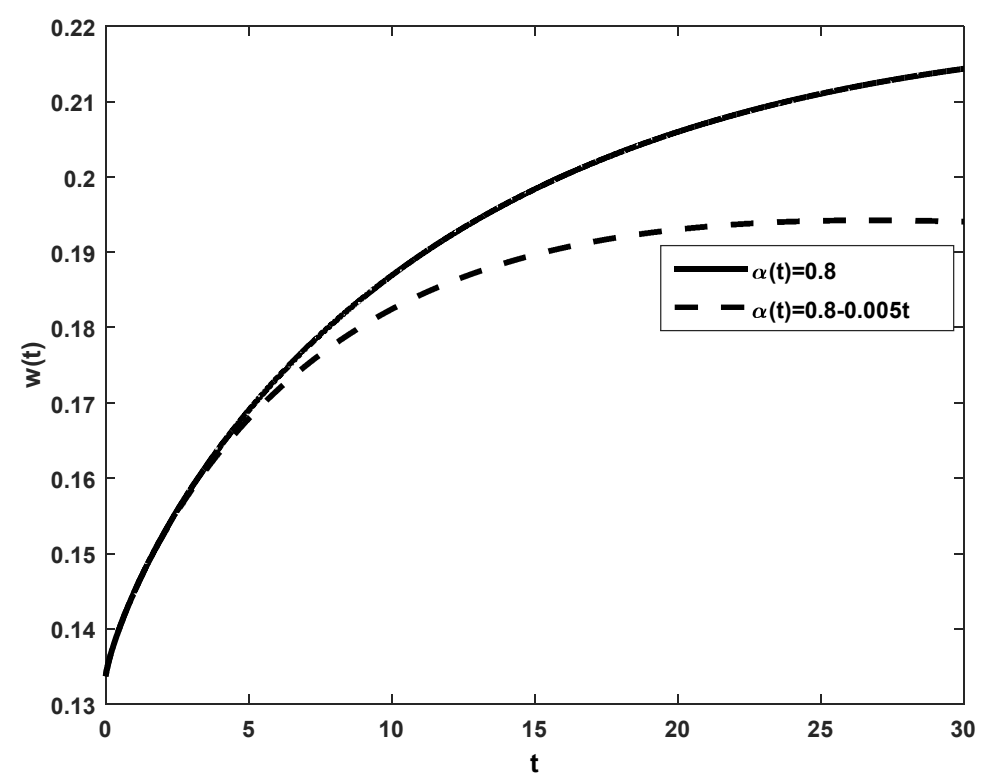

Fig.7: The solution of $\mathrm{w}(\mathrm{t})$ at $\alpha(t)=0.8$ (the solid line) and at $\alpha(t)=0.8-0.005 t$ (dashed line).

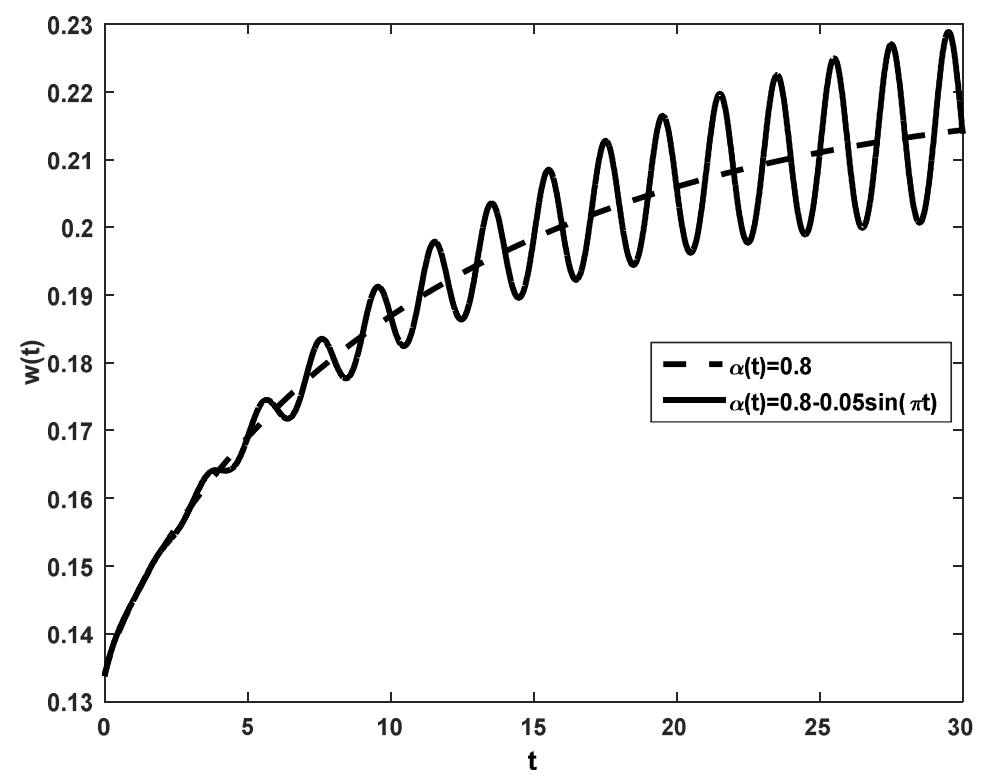

Fig. 8: The solution of $\mathrm{w}(\mathrm{t})$ at $\alpha(t)=0.8$ (the dashed line) and at $\alpha(t)=0.8-0.05 \sin (\pi t)$ (solid line).

In Figs. 1, 3, 5, 7 we take the variable-order fractional $\alpha_{i}(t)=0.8-0.005 t$ means the memory of the system is a decreasing function so the system behavior is slower with time.

In figs. 2, 4, 6, 8 we take the variable-order fractional $\alpha_{i}(t)=0.8-0.05 \sin (\pi t)$ means the memory of the system is a periodic function so the system behavior is a periodic. 


\section{Conclusion}

This paper introduced characterizing the memory property of smoking habit model. we used the numerical results to show that according to the formula of the memory of the system such as a decreasing function or a periodic function the behavior of the system has the same properties of the memory function.

\section{References}

El-Sayed, A.M.A., M. Khalil, A.A.M. Arafa and Amaal Sayed, 2016. Numerical behavior of a fractional order dynamical model of RNA silencing”. International Journal of Scientific World, 4(2):52-56.

El-Sayed, A.M.A., A.A. Arafa, M. Khalil and Amaal Sayed. 2017. A. Backward Bifurcation in a Fractional Order Epidemiological Model. An international journal of progress in fractional differentiation and application, 3(4): 281-287.

Khalil M., A.A. Arafa and Amaal Sayed, 2018. A variable fractional order network model of Zika virus". Journal of Fractional Calculus and Applications, 9(1), 204-221.

Arafa A.A.M., M. Khalil and A. Sayed, 2019. A Non-Integer Variable Order Mathematical Model of Human Immunodeficiency Virus and Malaria Co-infection with Time Delay" complexity, Hindawi, volume 2019, article ID 4291017.

Guerrero F., F.J. Santonja and R.J. Villanueva, 2011. "Analysing the effect of Spanish smoke-free legislation of year 2006: a new method to quantify its impact using a dynamic model, Int. J. Drug Policy, 22: 247-251.

Turski A.J., B. Atamaniuk and E. Turska, 2002. Fractional derivative analysis of Helmholtz and paraxial-wave equations". arXiv preprint physics/0203047.

Zaman, G. 2011. Qualitative behavior of giving up smoking models. Bulletin of the Malaysian Mathematical Sciences Society, 34(2):403-415.

Garsow Castillo, Carlos, Guarionex Jordan-Salivia, and Ariel Rodriguez-Herrera, 1997. "Mathematical models for the dynamics of tobacco use, recovery and relapse.

Pinto, C.M.A. and J.A.T. Machado, 2013. Fractional model for malaria transmission under control strategies", Computers and Mathematics with Applications, 66(5): 908-916.

$\mathrm{Xu}, \mathrm{Y}$. and Z. He, 2013. Existence and uniqueness results for Cauchy problem of variable-order fractional differential equations, J. Appl. Math. Comput., 43(1-2): 295-306.

Guerrero F., F.J. Santonja, R.J. Villanueva, 2011. Analysing the effect of Spanish smoke-free legislation of year 2006: a new method to quantify its impact using a dynamic model, Int. J. Drug Policy 22:247-251. 